\title{
Selected Risk Factors' Contribution to State-Level Incidence of Diagnosed Diabetes, 2005-2007
}

\author{
Lawrence Barker ${ }^{*}$, Edward Tierney, Andrew Lanza and Karen Kirtland
}

Centers for Disease Control and Prevention, USA

\begin{abstract}
Introduction: Differences in incidence of diabetes and prevalence of risk factors for diabetes exist among states. It is unknown how much of this variability in incidence of diagnosed diabetes is due to variability in risk factor prevalence. We investigate the contribution of selected risk factors to state level incidence of diagnosed diabetes.

Materials and Methods: Using 2005-2007 data from the Behavioral Risk Factor Surveillance System, we conducted two logistic regressions, both with incident case status as dependent variable. One model considered only state of residence as an independent variable. The other added: age; sex; race/ethnicity; education; inactive lifestyle; and obesity. We compared adjusted and unadjusted odds of incident diabetes among states, and calculated excess risk.

Results: Adjusted and unadjusted odds of incident diabetes were similar. Sensitivity analyses showed that this differed little if we used data from earlier years or if we included income or insurance as a risk factor. In most states, the excess risk associated with risk factors was less than $30 \%$.

Discussion: Factors other than age, sex, race/ethnicity, education, inactivity, and obesity (i.e., established risk factors for diabetes) might substantially influence the differences in state incidence rates. These factors' identities are unknown. If these factors are identified and modifiable, states might use them to reduce between-state disparities in diabetes incidence.
\end{abstract}

Keywords: Diabetes, incidence, risk factors, state level analyses.

\section{INTRODUCTION}

Diabetes is associated with increased risk of heart disease [1], vision loss [1], kidney disease [1], and lower limb amputation [1]. Diabetes is costly in terms of both direct and indirect costs [2]. Remission from diabetes is rare and usually temporary [3]; for the overwhelming majority, diabetes is a lifetime condition.

While incidence of diagnosed diabetes differs dramatically among states within the United States [4], the prevalence of risk factors for developing diabetes, which influence the incidence of diabetes, also differs among states. For example, in 2008, the estimated state-level prevalence of obesity, a major risk factor for diabetes, ranged from $18.5 \%$ (Colorado) to $32.8 \%$ (Mississippi) [5]. Figs. (1-3) display, respectively, 2006 state level incidence of diabetes and state level prevalence of obesity and physical inactivity (some states reported on in the Figures were not included in this analysis, due to different methods.) It is unknown how much of the variability in state incidence of diabetes is due to variability among states in prevalence of risk factors, such as obesity and sedentary lifestyle, and how much is due to other more difficult to measure factors, such as variability in access to care of prediabetes or income inequality [6]. Here, we investigate the contribution of selected risk factors to state level risks of incidence of diagnosed diabetes.

*Address correspondence to this author at the 2877 Brandywine Road Mailstop K-10, Atlanta GA, USA; Tel: 770-488-4403; Fax: 770-488-8634;

E-mail:1sb8@cdc.gov

\section{MATERIALS AND METHODS}

\section{Data Source}

The Behavioral Risk Factor Surveillance System (BRFSS) is a state-based system of annual cross-sectional health surveys. The BRFSS assesses key behavioral risk factors and chronic conditions in non-institutionalized U.S. adults aged $\geq 18$ years. Participants were selected from civilian residents with land line telephones by random digit dialing [7].

BRFSS respondents were asked, "Have you ever been told by a doctor that you have diabetes?" Women who reported only having diabetes during pregnancy were not counted as having diabetes. Current age and age at time of diagnosis were self-reported. Physical activity was assessed by the answer to the question, "During the past month, other than your regular job, did you participate in any leisure time physical activity?" We calculated body mass index (BMI) as the ratio of self reported weight $(\mathrm{kg})$ (participants were asked, "About how much do you weigh without shoes?") divided by self-reported height (participants were asked, "About how tall are you without shoes?") squared $\left(\mathrm{m}^{2}\right)$. Sociodemographics (age, race/ethnicity, sex, and education) were self-reported.

\section{Analysis}

We based our analysis on the combined 2005 through 2007 BRFSS. All analyses were weighted, to represent the population. Among those self-reporting diabetes, we calculated diagnosed diabetes duration by subtracting 




Fig. (1). Estimated state level incidence of diabetes in the United States, 2006. (Source: http://apps.nccd.cdc.gov/DDTSTRS/State SurvData.aspx).

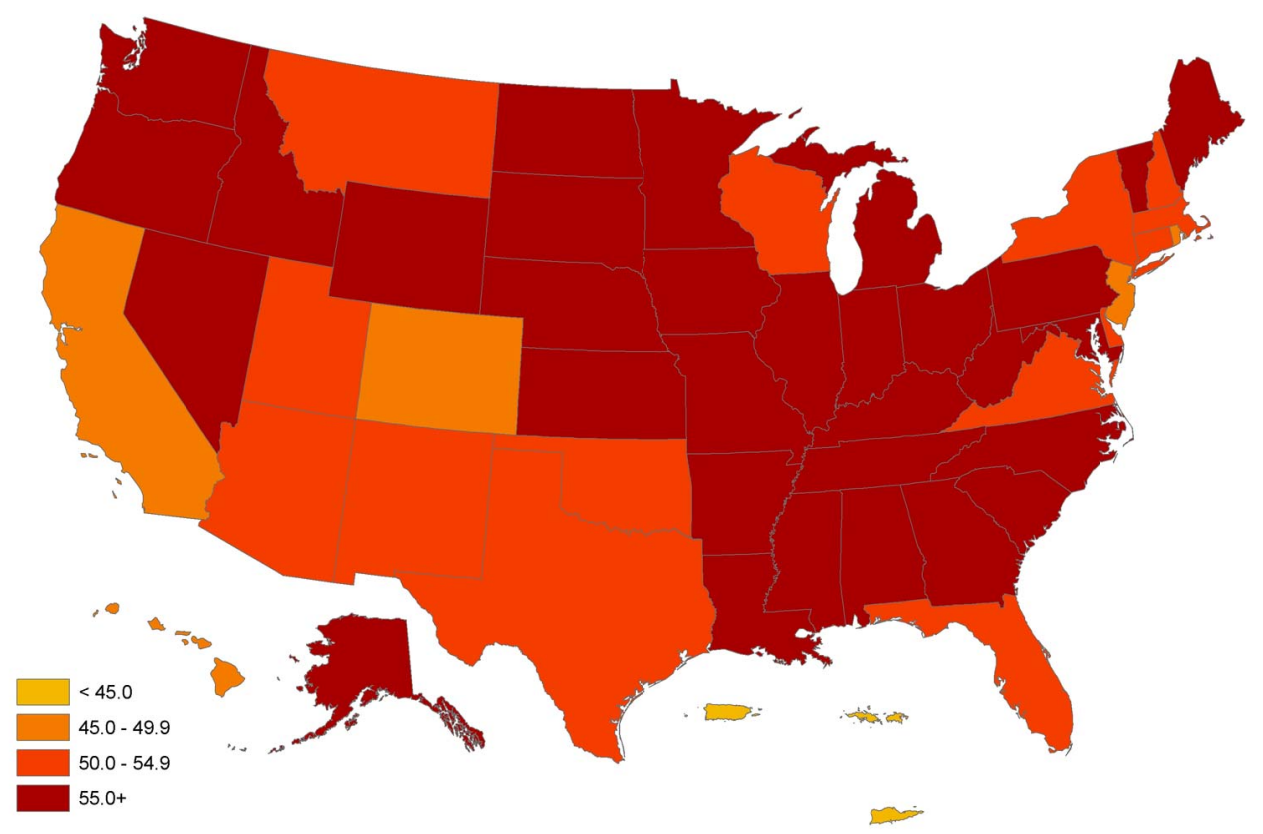

Fig. (2). Estimated state level prevalence of obesity in the United States, 2006. (Source: http://apps.nccd.cdc.gov/DDTSTRS/StateSurv Data.aspx). 


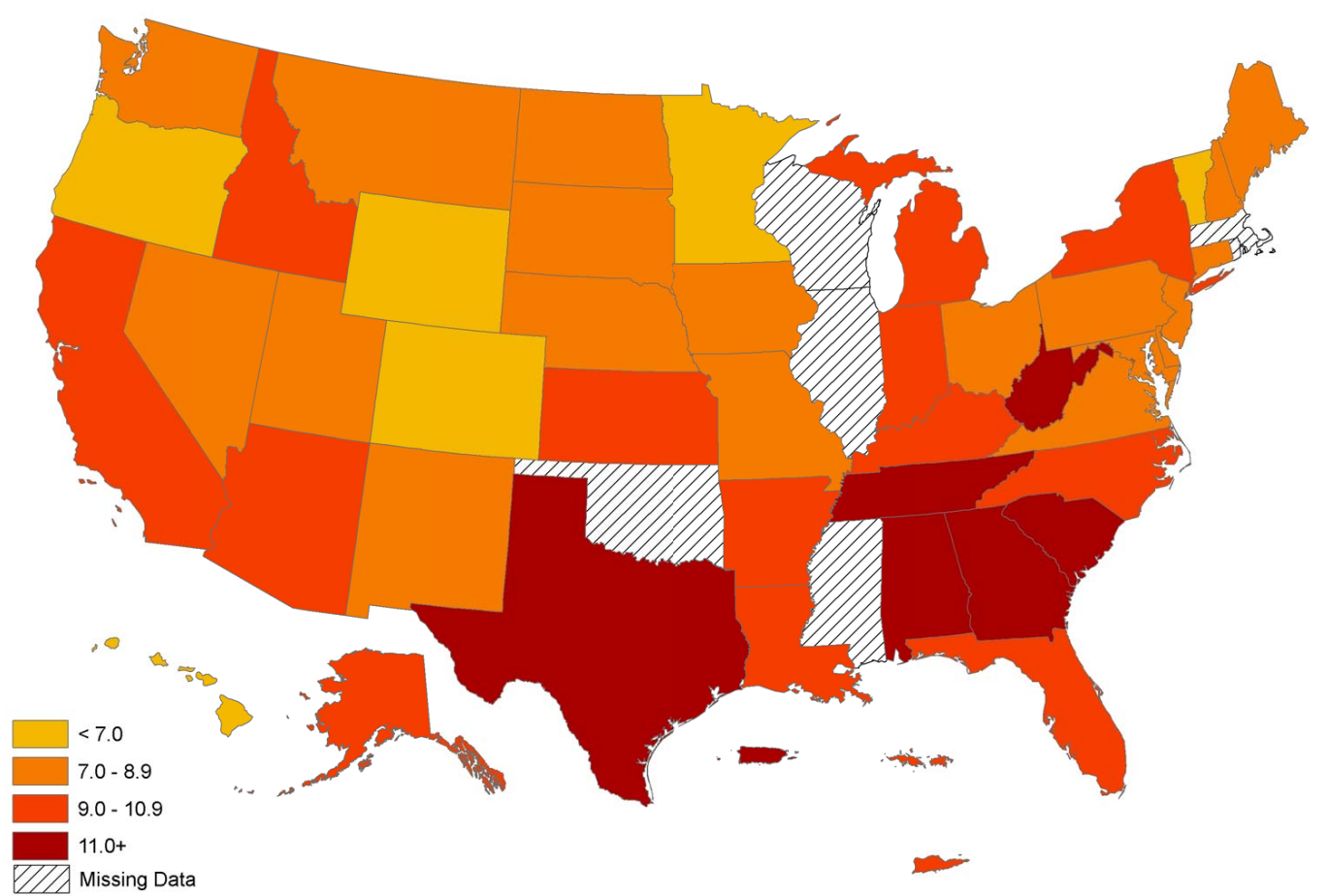

Fig. (3). Estimated state level prevalence of physical inactivity in the United States, 2006. (Source: http://apps.nccd.cdc.gov/DDTSTRS/State SurvData.aspx).

self-reported age at diagnosis from self-reported age at time of interview. Those reporting zero years since diagnosis were considered incident cases. Those not reporting diabetes were considered non-incident cases. Those reporting diagnoses of diabetes two or more years ago were excluded from all analyses, because they could not be incident cases. For those who self-reported diagnosis one year ago, we multiplied the existing weight by 0.5 . This was done because, under the assumption that diagnosis of diabetes is uniformly distributed over the year, the probability that a person whose age at time of interview was one more than their age at time of diagnosis of diabetes is 0.5 .

We conducted two logistic regressions, both with incident case status as dependent variable. In one model, we considered only state of residence as an independent variable; we chose Minnesota, the state that Kirtland et al. [4] found to have the lowest unadjusted incidence (5.0 per $1000)$, as reference. In the other, the independent factors considered were: state of residence; age ( $\leq 44,45-65, \geq 65$ years); sex; race/ethnicity (Hispanic, non-Hispanic white, non-Hispanic black, multi-racial, other); education (did not graduate high school, graduated high school, attended but did not complete college or technical school, completed college or technical school); report of engaging in no leisure time physical activity in the last month; and obesity, defined by self-reported BMI $\geq 30 \mathrm{~kg} / \mathrm{m}^{2}$. We omitted from both analyses those cases with any missing data in any factors used in the analysis. Thus, the same individuals' responses were used in both models. To estimate what excess risk, compared with the reference state, for incident diabetes was associated with selected covariates, the percentage change of the odds ratio with and without the covariates using the expression $[(\mathrm{OR} 1$ - OR2)/(OR1 - 1.0)] $\times 100 \%$ was calculated, where OR1 represents OR derived from the basic model, OR2 represents odds ratio after adjusting for additional covariates, and 1.0 represents odds ratio when no excess risk exists

As a sensitivity analysis, we repeated the analysis using data from 2002 - 2004. This was considered a sensitivity analysis because we were unsure if the results were stable over time. As a further sensitivity analysis, we repeated the analysis including annual household income $(<\$ 15000$, $\$ 15000$ - $<\$ 25,000, \$ 25000-<\$ 35,000, \$ 35,000-<\$ 50,000$, $\geq \$ 50,000)$. We considered the model using income a sensitivity analysis because of the income question's high non-response rate $(13.8 \%)$. We repeated the analysis including insurance coverage, in addition to age, education, inactivity, obesity, race/ethnicity, sex. This was considered a sensitivity analysis because insurance coverage was measured as of the time of the survey, and not at the time the respondent's diabetes was diagnosed.

\section{RESULTS}

Not all states gathered diabetes data in all years. We report results for those states that gathered diabetes data in at least two out of three years, 2005 - 2007 (Table 1). Our total, 
Table 1. Odds Ratios of Incidence of Diabetes Per State, 2005-2007, and Excess Risk



unweighted annual sample size, after excluding those with missing data, averaged 747,012.

Table 1 reports both unadjusted and adjusted odds ratios. Our results can be interpreted by noting that greater odds ratios, whether adjusted or not, are associated with greater incidence of diagnosed diabetes. Ohio had the smallest odds ratio (1.22 unadjusted, 1.07 adjusted), while West Virginia had the largest (2.71 unadjusted, 2.24 adjusted). For many 
states, adjusted odds ratios were closer to 1.00 than unadjusted. However, this was not universal; for example, in Colorado (a state with a low prevalence of obesity), adjustment increased the odds ratio from 1.18 to 1.33 . In general, adjusted and unadjusted ratios were similar.

Table 1 reports excess risks. Positive excess risks means that, after adjusting for age, education, inactivity, obesity, race/ethnicity, and sex (individual, and not state, level adjustment), state disparities decreased. Negative excess risks means that, after the same adjustment, state disparities increased (for example, a state with a lower prevalence of obesity might have its disparity increased by adjusting). The absolute value of excess risks varied from essentially zero to more than $100 \%$ (excess risks can exceed $100 \%$ when the unadjusted odds ratio is larger than one but the adjusted odds ratio is less than one).

The 2002-2004 results were, broadly, similar (Table 2). The results accounting for income were also broadly similar to those that did not account for income (Table 1). Although we do not report the odds ratios for the model that included insurance, all state odds ratios considering insurance are within \pm 0.04 of the same state not considering insurance.

\section{DISCUSSION}

Table 1 shows that, while adjustment for age, education, inactivity, race/ethnicity, and sex makes some difference, adjusted and unadjusted ratios are substantially similar. For all but a few states, age, education, inactivity, obesity, race/ethnicity, and sex account for less than $30 \%$ of state disparity (32 of the 41 absolute excess risks were less than $30 \%$ ). These factors explain more than half the disparity only in the states of Colorado, Hawaii, Ohio, and Utah, all of which differ from the remaining states in some substantial manner (e.g. low prevalence of obesity in Colorado and Hawaii, substantially higher proportion of persons of 'other' race/ethnicity and lower proportion of non-Hispanic whites than other states in Hawaii, high prevalence of obesity in Ohio, low prevalence of obesity, tobacco use, and alcohol use in Utah). Of note, all states with negative excess risk had a prevalence of obesity below the national median.

While all factors in the more complicated model are significant ( $\mathrm{p}<0.01$ for all factors), the addition of multiple well-known risk factors for diabetes in the United States to the model (age, education, inactivity, obesity, race/ethnicity, sex ) resulted in relatively little change of the odds ratios associated with states. In the majority of the states, this addition accounted for less than a third of the excess risk. Thus, factors other than differences in state demographics (age, education, race/ethnicity) and prevalence of obesity and sedentary lifestyle, two important risk factors for diabetes and the focus of many diabetes prevention initiatives, might account for much of the differences among states in incidence of diagnosed diabetes. Although individual level income explains some additional difference, it contributes relatively little (with the possible exceptions, depending on how large a change one considers as 'contributing relatively little', of the states of Alaska, Arizona, Connecticut, and Nevada); insurance coverage at the time of the survey contributes less than did income. Due to limitations of our data source, we are unable to determine what the factors that cause the remainder of the difference might be.

It is unclear how much the observed differences among state incidences of diagnosed diabetes represent actual differences in incidence of diabetes (both diagnosed and undiagnosed) and how much they represent differences in rates of diagnosis among those with diabetes. Residents in states with poorer access to care could also have more undiagnosed diabetes. It is also possible that states differ in the extent to which obesity and sedentary lifestyle are underreported due to social desirability bias, which could influence our results. We recommend that public health officials in states with larger odds ratios consider the possibility that their states might have a high incidence of diabetes, and that all states consider the possibility that their state might have substantial undiagnosed diabetes. Public health responses to these should differ. States that might have a high incidence should consider programs to decrease the incidence of diabetes. While culturally appropriate interventions should include efforts directed at decreasing obesity and sedentary lifestyle, the results herein suggest that other factors, possibly including community-level poverty (we measured individual income, not community level), access to and level of care for those with prediabetes, and other appropriate issues might also be considered. States that might have high rates of undiagnosed diabetes should consider encouraging providers to test persons at high risk for diabetes. While universal screening for diabetes is undesirable, targeted screening can be effective [10].

Our results are consistent with Kirtland et al. [4], who found greater incidence of diagnosed diabetes in the southeast and in some western states. They are also consistent with Gregg et al. [8], who found greater prevalence of diagnosed diabetes in Appalachia, the southeast, and in some western states and with Barker et al. [9], who found residence in the Appalachian region to be a risk factor for diabetes even after controlling for obesity, sedentary lifestyle, age, poverty, and other selected risk factors.

Kirtland et al.'s [4] estimates of state incidence of diabetes include unadjusted and age adjusted estimates only. This analysis adds to the literature by providing estimates adjusted by other demographics in addition to age. To the best of the authors' knowledge, this work is the first comparison of state incidences adjusting for multiple risk factors for diabetes. However, other studies have examined the relationship between geographic and demographic risk factors for other diseases. For example, Klassen et al. [11] found that geographic clustering of prostate cancer in Maryland looked substantially different with and without adjustment for risk factors. Liao et al. [12] showed that demographics explained approximately $72 \%$ of the difference between 'stroke belt' states and the rest of the US in the prevalence of non-fatal stroke.

Our sensitivity analyses suggest that our results might be relatively stable over time (odds ratios from state only and state plus risk factors differed little in either 2002-2004 or 2005-2007) and that inclusion of income and insurance in the model made relatively little difference (odds ratios 
Table 2. Results of 2002-2004 Analysis

\begin{tabular}{|c|c|c|}
\hline United States State & $\begin{array}{c}\text { Odds Ratio and } 95 \% \text { Confidence Interval. Independent } \\
\text { Variable: State of Residence Only }\end{array}$ & $\begin{array}{l}\text { Adjusted Odds Ratio and } 95 \% \text { Confidence Interval. } \\
\text { Independent Variables: State of Residence, Age, } \\
\text { Education, Inactivity, Obesity, Race/Ethnicity, Sex }\end{array}$ \\
\hline Alabama & $2.02(1.46-2.79)$ & $1.60(1.15-2.23)$ \\
\hline Alaska & $0.89(0.50-1.58)$ & $0.79(0.44-1.41)$ \\
\hline Arizona & $1.19(0.82-1.72)$ & $1.14(0.78-1.66)$ \\
\hline Arkansas & $1.36(0.99-1.98)$ & $1.17(0.84-1.62)$ \\
\hline California & $1.62(1.15-2.28)$ & $1.39(0.98-1.97)$ \\
\hline Colorado & $0.93(0.64-1.34)$ & $1.01(0.70-1.47)$ \\
\hline Connecticut & $0.90(0.63-1.27)$ & $0.89(0.62-1.26)$ \\
\hline Delaware & $1.29(0.89-1.86)$ & $1.18(0.81-1.71)$ \\
\hline District of Columbia & NA & NA \\
\hline Florida & $1.56(1.11-2.20)$ & $1.29(0.91-1.82)$ \\
\hline Georgia & $1.40(1.01-1.95)$ & $1.24(0.89-1.74)$ \\
\hline Hawaii & $1.47(0.92-2.34)$ & $1.14(0.69-1.90)$ \\
\hline Idaho & $1.42(1.04-1.96)$ & $1.45(1.05-1.99)$ \\
\hline Indiana & $1.75(1.23-2.49)$ & $1.56(1.09-2.23)$ \\
\hline Iowa & $1.30(0.93-2.09)$ & $1.21(0.87-1.69)$ \\
\hline Kentucky & $1.48(1.07-2.05)$ & $1.34(0.96-1.87)$ \\
\hline Louisiana & $1.46(1.06-1.99)$ & $1.18(0.86-1.63)$ \\
\hline Maine & $1.49(1.04-2.14)$ & $1.47(1.03-2.12)$ \\
\hline Minnesota & Reference & Reference \\
\hline Missouri & $1.42(1.01-1.98)$ & $1.27(0.91-1.79)$ \\
\hline Montana & $0.99(0.69-1.43)$ & $0.97(0.67-1.39)$ \\
\hline Nevada & $1.10(0.71-1.69)$ & $0.98(0.63-1.52)$ \\
\hline New Hampshire & $1.15(0.83-1.60)$ & $1.18(0.85-1.64)$ \\
\hline New Jersey & $1.23(0.91-1.65)$ & $1.07(0.79-1.45)$ \\
\hline New Mexico & $0.93(0.67-1.29)$ & $0.80(0.57-1.14)$ \\
\hline New York & $1.35(0.95-1.93)$ & $1.18(0.83-1.68)$ \\
\hline North Carolina & $1.44(1.06-1.94)$ & $1.26(0.93-1.71)$ \\
\hline North Dakota & $1.08(0.67-1.74)$ & $0.99(0.61-1.60)$ \\
\hline Ohio & $1.56(1.06-2.28)$ & $1.38(0.94-2.03)$ \\
\hline Oregon & NA & NA \\
\hline Pennsylvania & $1.34(0.96-1.88)$ & $1.17(0.83-1.64)$ \\
\hline South Carolina & $1.73(1.28-2.33)$ & $1.50(1.10-2.04)$ \\
\hline South Dakota & $1.14(0.82-1.58)$ & $1.06(0.76-1.47)$ \\
\hline Tennessee & $1.85(1.31-2.63)$ & $1.66(1.16-2.36)$ \\
\hline Texas & $1.93(1.42-2.63)$ & $1.68(1.23-2.29)$ \\
\hline Utah & $1.04(0.73-1.48)$ & $1.20(0.84-1.71)$ \\
\hline Vermont & $1.03(0.74-1.44)$ & $1.06(0.76-1.49)$ \\
\hline Virginia & $1.26(0.82-1.77)$ & $1.19(0.84-1.68)$ \\
\hline Washington & $1.44(1.09-1.90)$ & $1.43(1.08-1.90)$ \\
\hline West Virginia & $2.02(1.45-2.81)$ & $1.71(1.22-2.39)$ \\
\hline Wyoming & $1.36(0.95-1.95)$ & $1.32(0.93-1.87)$ \\
\hline
\end{tabular}


changed relatively little when income was added to the model). While income is a measure of socioeconomic status, which has an impact on diabetes prevalence [13], it is possible that education might have contained enough information about socioeconomic status for income to provide relatively little additional information beyond that included in education.

This report is subject to several limitations. First, BRFSS data are self-reported and subject to non-reporting, social desirability, recall, and other biases. Similarly, BRFSS excludes households without land-line telephones, which introduces additional bias. Second, we could only consider diagnosed diabetes. Our data source did not let us distinguish between type 1 and type 2 diabetes, although between 90 and $95 \%$ of the cases of diabetes are type 2 [14]. Further, we were unable to distinguish development of diabetes from initial diagnosis, and type 2 diabetes can remain undetected for years. It is unknown how the percent of cases of diabetes that are undetected vary among states. While it is plausible that the impact of risk factors might vary among states, we did not consider such state of residence by risk factor interactions. Had such interactions been found, they would have made our results difficult to present or interpret. Finally, we were unable to determine how long respondents had lived in a particular state. Recent and long-term residents of a state might have different incidences of diabetes.

Much state and national-level diabetes surveillance and goal setting [15] has focused on prevalence, not incidence. This is understandable, because prevalence is easier to measure than incidence. Incident cases are rare and prevalence is the primarily driver for diabetes' social and economic costs. In the early years of state diabetes programs and the BRFSS, prevalence was the only measure available for determining the success of state programs. With the advent of the optional diabetes module in the BRFSS and inclusion of data on age at time of diagnosis, incidence data became available to states. However, most states' sample sizes precluded any meaningful analyses. In more recent years, sample sizes in most states have increased. The increased sample sizes and the combining of multiple years of data now allow most states to evaluate diagnosed diabetes incidence. In fact, national diabetes goal setting has, in recent years, moved from prevalence to incidence measures [16].

While efforts to decrease obesity and sedentary lifestyle (two strong, modifiable risk factors for type 2 diabetes) to reduce diabetes incidence should continue, we should consider the possibility that other factors, perhaps difficult to measure, might also play a substantial role. If identified and found to be modifiable, these risk factors could play a role in reducing disparities among states. However, monitoring these factors might require state-level surveillance of diabetes beyond what is currently conducted. The identification of these factors must be left to future research, although their identification might provide a key to the reduction of diabetes in the United States. The overall ageadjusted death rate for people with diabetes is twice that for people without diabetes and it is two to four times that for people with diabetes who die of heart disease [17]. A 1994 Medicare study found that the age-, sex-, and race/ethnicityadjusted state-specific prevalence and incidence rates of diabetes varied 2-3 times (from 18.8/100 to $8.9 / 100$ for prevalence and from 3.8/1000 to $1.4 / 1000$ for incidence). However, despite this wide range in state-specific prevalence and incidence rates, among those with diagnosed diabetes, the mortality rates in 1994 in high-prevalence and lowprevalence states were similar (from 107.8/1000 to 81.9/1000) [18]. Regardless of state, those with diabetes face many complications of this disease, including mortality. Reducing excess incidence of diabetes will help states reduce one cause of premature mortality among their populations.

Finally, these results are of limited direct use to a nonUnited States audience. However, similar methods are applicable in any nation that has similar data available at any subnational level of geographic discrimination. These methods could be used by other nations to determine if subnational geographic disparities are or are not primarily attributable to such risk factors as obesity, age, poverty, and whatever risk factors for diabetes that are appropriate for the nation in question.

The contents of this paper are solely the responsibility of the authors and do not necessarily represent the official positions of the Centers for Disease Control and Prevention.

\section{AUTHORS CONTRIBUTION}

LB conceived the question behind this manuscript and wrote most of the manuscript. ET provided the statistical analysis. AL reviewed and commented on the manuscript, and provided expertise in state diabetes programs. KK reviewed and commented on the manuscript and provided expertise on incidence.

\section{ACKNOWLEDGEMENT}

None declared.

\section{CONFLICT OF INTEREST}

None declared.

\section{ABBREVIATIONS}

\author{
BRFSS $=$ Behavioral Risk Factor Surveillance System \\ BMI = Body Mass index

\section{REFERENCES}

[1] Nathan DM. Long-Term Complications of Diabetes Mellitus. NEJM 328 1993; 1676-85.

[2] Hogan P, Dall T, Nikolov P. Economic costs of diabetes in the US in 2002. Diabetes Care 2003; 26: 917-32.

[3] Sjostrom L, Lindroos AK, Peltonen M, et al. Lifestyle, diabetes, and cardiovascular risk factors 10 years after bariatric surgery. NEJM 2003; 351: 2683-93.

[4] Kirtland KA, Li YF, Geiss LS, Thompson TJ. State-Specific Incidence of Diabetes Among Adults -Participating States, 19951997 and 2005-2007. MMWR 2008; 57(43): 1169-73.

[5] Centers for Disease Control and Prevention. U.S. Obesity Trends by State 1985-2008. www.cdc.gov/obesity/data/trends.html (referenced August 17, 2011)

[6] Wilkinson R, Pickett K. The Spirit Level: Why greater equality makes societies stronger. London: Allen Lane 2009.

[7] Holtzman D. The behavioral risk factor surveillance system. In Blumenthal DS, Di Clemente RJ, Eds. Community-Based Health Research: Issues and Methods. New York: Springer 2003; 115-31.

[8] Gregg EW, Kirtland KA, Cadwell BL, et al. Estimated CountyLevel Prevalence of Diabetes and Obesity - United States, 2007. MMWR 2009; 58(45); 1259-63.

[9] Barker L, Crespo R, Gerzoff R, Denham S, Shrewsberry M, Cornelius-Averhart D. Residence in a distressed county in 
Appalachia as a risk factor for diabetes. Prev Chronic Dis 2010; 7: 5.

[10] O'Connor PJ, Rush WA, Cherney LM, Pronk NP. Screening for diabetes mellitus in high-risk patients: cost, yield, and acceptability. Effective Clinical Practice 2001; 4: 271-7.

[11] Klassen AC, Kulldorffand M, Curriero F. Geographical clustering of prostate cancer grade and stage at diagnosis, before and after adjustment for risk factors. Int $\mathrm{J}$ Health Geogr 2005; 4 : 1 .

[12] Liao Y, Greenlund K, Croft J, Keenan L, Giles W. Factors Explaining Excess Stroke Prevalence in the US Stroke Belt. Stroke 2009; 40: 3336-41.

[13] Connolly V, Unwin N, Sherriff P, Bilous R, Kelly W. Diabetes prevalence and socioeconomic status: a population based study showing increased prevalence of type 2 diabetes mellitus in deprived areas. J Epidemiol Community Health 2000; 54: 173-7.
[14] Centers for Disease Control. National Diabetes Fact Sheet. www.cdc.gov/diabetes/pubs/estimates05.htm (referenced August 17, 2011)

[15] US Department of Health and Human Services. Healthy People 2010. www.healthypeople.gov/Document/tableofcontents.htm\#vol ume1 (referenced August 17,2011)

[16] US Department of Health and Human Services. Healthy People 2020. www.healthypeople.gov/2020/topicsobjectives2020/objectiv eslist.aspx?topicId $=8$ (referenced August 17, 2011)

[17] Geiss LS, Herman WH, Smith PJ. Mortality in non-insulindependent diabetes. In: I. Harris MI, Cowie CC, Stern MP, Boyko EJ, Reiber EG and Bennet PH, Editors, Diabetes in America (2nd ed), US Government Printing Office, Washington, DC (1995), (NIH publication No. 95-1468) 1995; pp. 233-57.

[18] Tierney EF, Cadwell BL, Thompson TH, et al. Reductions in excess mortality rates among people with diabetes by selected cause of death. J Diabetes Complicat 2009; 23(2): pp. 77-82.

C Barker et al.; Licensee Bentham Open.

This is an open access article licensed under the terms of the Creative Commons Attribution Non-Commercial License (http://creativecommons.org/licenses/by-nc/3.0/) which permits unrestricted, non-commercial use, distribution and reproduction in any medium, provided the work is properly cited. 